\title{
Hypothyroidism: A Silent Killer in Young Patients
}

\author{
Mariela Melissa Rivera Agosto ${ }^{\mathrm{a}}$, , Fermin Lopez Rivera $^{\mathrm{b}}$, Hernan Gonzalez Monroig ${ }^{\mathrm{b}}$, \\ Paula Jeffs Gonzalez ${ }^{c}$, Fernando Abreu Gonzalez ${ }^{b}$
}

\begin{abstract}
Hypothyroidism is considered by many as a "great mimicker" of many common complaints in the emergency department (ED). Thyroid disorders pose a significant threat when prolonged or in the acute presentation, and are potentially serious disorders with symptoms that range from non-specific constitutional to subtle or frank neuropsychiatric symptoms. Untreated disease can lead to myxedema crisis or coma, a life-threatening presentation that is rarely identified in young male patients and carries by itself a high risk of mortality, even in cases that undergo proper medical management. We present a case of a 26 -year-old non-smoker Hispanic man with a medical history of hypothyroidism since the age of 13 , and bronchial asthma. In a period of 3 - 4 months, he developed generalized malaise, fatigue, cold intolerance and unintentional weight loss. He visited the ED after developing acute abdominal pain associated with non-bloody watery diarrheas. At the initial evaluation, vital signs revealed hypotension, borderline low heart rate, and hypoglycemia. On physical examination, he looked pale, appeared lethargic, with facial puffiness, macroglossia and bilateral hyporeflexia with delayed relaxation on muscle stretch reflexes. The laboratory results showed a thyroid stimulating hormone (TSH) of $314.75 \mathrm{uIU} / \mathrm{mL}$. There was also low sodium and elevated creatinine and hepatic enzymes levels. Abdominopelvic CT scan incidentally revealed a large pericardial effusion that was later confirmed with echocardiogram. A diagnosis of myxedema crisis was established, prompt initiation of intravenous hydrocortisone, levothyroxine and triiodothyronine therapy was administered, and he was transferred to another institution for management of the pericardial effusion. This case raised concerns about the incidence where thyroid disease presentations are not identified and where patients fail to receive adequate medical therapy. We as primary care physicians should encourage patients to adhere to medical therapy and the recommended follow-up instructions to avoid catastrophic complications such as myxedema crisis.
\end{abstract}

Manuscript submitted October 16, 2017, accepted November 10, 2017

${ }^{a}$ Department of Internal Medicine, UPR Medical Sciences, San Juan, Puerto Rico

bDepartment of Internal Medicine, San Juan City Hospital, San Juan, Puerto Rico

'Department of Endocrinology, San Juan City Hospital, San Juan, Puerto Rico ${ }^{\mathrm{d} C o r r e s p o n d i n g ~ A u t h o r: ~ M a r i e l a ~ M e l i s s a ~ R i v e r a ~ A g o s t o, ~ D e p a r t m e n t ~ o f ~ I n t e r-~}$ nal Medicine, UPR Medical Sciences, Urb. La Esperanza C/2 N20 Vega Alta, Puerto Rico. Email: mariela_1324@hotmail.com

doi: https://doi.org/10.14740/jmc2942w
Keywords: Myxedema coma/crisis; Facial puffiness; Macroglossia; Bilateral hyporeflexia with delayed relaxation; Pericardial effusion

\section{Introduction}

Hypothyroidism is a clinical condition that results from inadequate synthesis of thyroid hormones and is considered a "great mimicker" of many common complaints found in the emergency department (ED). Mostly in patients unaware of their thyroid dysfunction or the severity of their condition [1], hypothyroidism may present as a potentially serious disorder, where patients may initially appear with non-specific constitutional and neuropsychiatric symptoms, and where infections and sepsis are the most common precipitation factors $[2,3]$. Untreated disease can lead to myxedema coma/ crisis, which is a life-threatening form of decompensated hypothyroidism most commonly in elderly woman and rarely identified in young male patients [4]. Despite of term myxedema coma, patients frequently do not present comatose, instead obtunded for which myxedema crisis may be an apt term. Because it is a rare and unrecognized disease, there are a few isolated cases reports and case series. The myxedema crisis carries by itself high mortality rates, approximately $25-60 \%$ even in cases that undergo proper medical management [5].

\section{Case Report}

We present a case of a 26-year-old Hispanic non-smoker male with medical history hypothyroidism since 13 years old and bronchial asthma who visited San Juan City Hospital (SJCH) urgency room due to acute abdominal pain since same day. He identified the pain at the left upper quadrant of the abdomen, described as stabbing, 7/10 in intensity, constant, non-radiating and without identifiable exacerbating or improving factors. He reported non-bloody watery diarrheas (3 - 4/day) associated to food intake, for the last week. He also noticed that over the past 3 - 4 months, he had developed generalized malaise, fatigue, cold intolerance, somnolence, mental slowing, headache, loss of appetite, unintentional and unquantified weight loss. He denied fever, chills, nauseas, vomiting, chest pain, shortness of breath, seizure or dysuria. He has history of allergic reaction to seafood, sulfa, aspirin and penicillin, described as edema. He was not taking any medications and denied a history of 
drug use or a family history of hereditary or familial disorder. On initial evaluation, vital signs revealed temperature of 36.9 ${ }^{\circ} \mathrm{C}$, blood pressure of 93/58 $\mathrm{mm} \mathrm{Hg}$ (MAP, $70 \mathrm{~mm} \mathrm{Hg}$ ), heart rate of 66 beats per minute, respiratory rate of 18 breaths per minute, oxygen saturation by finger pulse oximetry at $98 \%$, and blood finger dextro stick at $55 \mathrm{mg} / \mathrm{dL}$. Body mass index was $19.4 \mathrm{~kg} / \mathrm{m}^{2}$ (normal).

Physical examination showed pale appearance, hypoactive and lethargic, with mental slowing, barely keeping up a conversation. There was facial puffiness, swollen upper lip and macroglossia. On cardiac examination, he had regular rate and rhythm and normal S1 and S2 heart sounds; there was no JVD, murmurs, friction rub or palpable cardiac apical impulse. Lungs were clear bilaterally. On abdominal examination, abdomen was flat, had normoactive bowel sounds, soft and depressible to palpation, non-tender and had no hepatosplenomegaly. On extremities, there was no edema and had pulses $2+$ bilaterally throughout. Skin exam had mild pallor and normal skin turgor; there was no coarse hair, dry and/or cold skin. On neurological examination, mental status, Glasgow coma scale, scored $15 / 15$, motor system with normal tone and strength, and sensory was intact to pinprick. Deep tendon reflexes showed delayed relaxation on upper extremities and hyperactive $3+$ on both knee jerk and ankle reflexes.

The laboratory results were remarkable for thyroid stimulating hormone (TSH) of $314.75 \mathrm{uIU} / \mathrm{mL}$, low sodium level of $130.9 \mathrm{mmol} / \mathrm{L}$, elevated creatinine of $1.3 \mathrm{mg} / \mathrm{dL}$, aspartate aminotransferase (AST) of $87 \mathrm{U} / \mathrm{L}$ and alanine aminotransferase (ALT) of $103 \mathrm{U} / \mathrm{L}$. In view of patient's initial chief complaint, abdominopelvic CT scan done incidentally revealed a large pericardial effusion that was later confirmed by echocardiogram, which also showed dilated cardiomyopathy with a moderated size pericardial effusion and an ejection fraction of 30-35\% (Figs. 1 and 2).

Based on patient's presentation, physical examination,

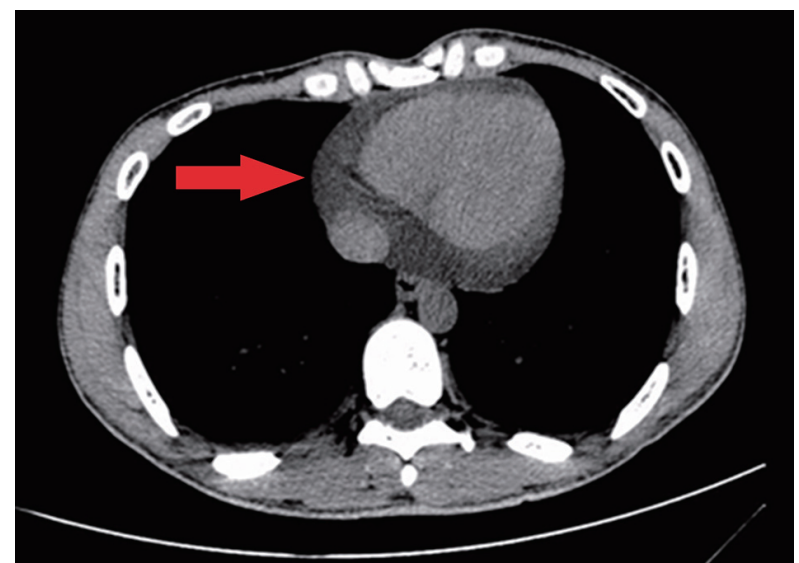

Figure 1. CT scan showing large pericardial effusion.

laboratories and evidence of a pericardial effusion, the diagnosis of myxedema crisis was established, a rare complication of untreated hypothyroidism. Once diagnosed for patient with myxedema crisis a prompt initiation of intravenous hydrocortisone, levothyroxine and triiodothyronine therapy was administered and he was transferred for management of the pericardial effusion.

\section{Discussion}

Myxedema crisis is a severe life-threatening complication associated with a mortality rate up to $60 \%$ despite proper medical management [5]. Hypothyroidism and myxedema crisis are more common in elderly and women patients [4]. Myxedema crisis is an extremely rare complication in young male patients. Significant predictors of mortality of myxedema crisis include
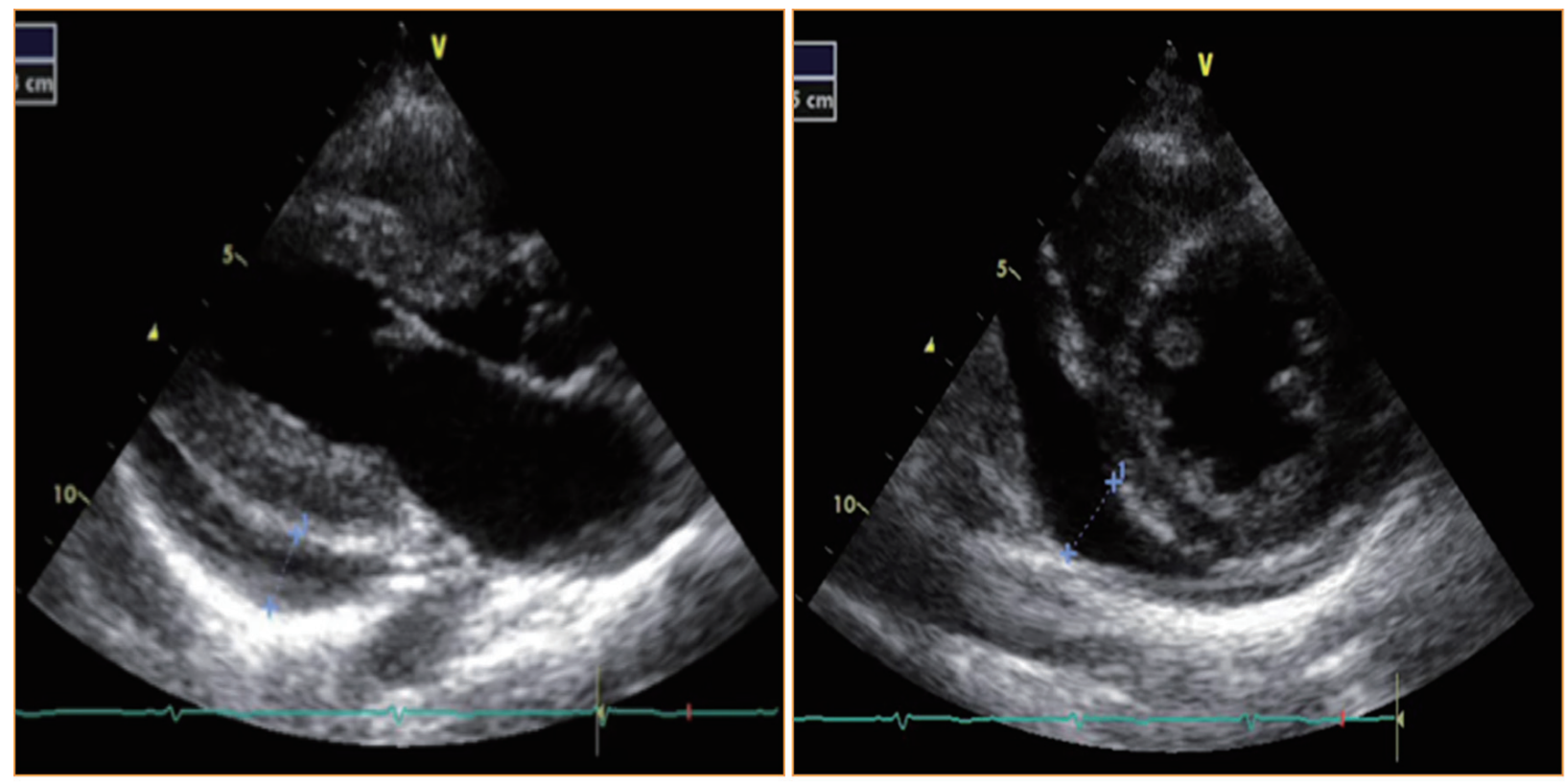

Figure 2. Echocardiogram showing pericardial effusion. 
hypotension and bradycardia at presentation. Low $\mathrm{T}_{3}$ leads to hypothermia and suppression of cardiac activity having consequences as respiratory depression, cardiogenic shock and coma [6]. These events are associated with poorer outcome in patients with history of cardiac disease. Severe untreated hypothyroidism is associated with heart failure, psychosis and coma. Congestive heart failure is rare in long-standing hypothyroidism. A study published in 2010 found that out of 33 patients with cardiomegaly, only six patients were found with a low left ventricular ejection fraction $(<40 \%)$ [7]. Recent studies have shown that the incidence of pericardial effusion in severe hypothyroidism ranges from $30 \%$ to $80 \%$ and that this complication can be prevented and/or regresses slowly with adequate levothyroxine therapy $[8,9]$.

\section{Conclusion}

There are few cases reported in recent literature related to myxedema crisis, as this is an exceedingly rare complication that is mostly seen in young and male patients. This case raised concerns about the incidence of people which are afflicted with thyroid disease in our population without proper medical management. Untreated severe hypothyroidism should be considered in patients who present with weakness, cold intolerance and/or altered mental status. Early diagnosis and adequate management improve patient's prognosis and decrease mortality. Primary care physicians should encourage patients to adhere to medical therapy and the recommended follow-up to avoid this catastrophic complication of hypothyroidism.

\section{Acknowledgments}

We wish to acknowledge the guidance and assistance of Karen Torres Gonzalez, MD, Yanery’s Agosto Vargas, MD, Samayra Miranda Rodriguez, MD, Michelle Mangual Garcia, MD and Christian E. Schenk, MD in preparation of this article.

\section{Financial Support}

No source of financial support to disclose.

\section{Conflict of Interest}

We have no conflict of interest to declare.

\section{References}

1. Miller KJ, Parsons TD, Whybrow PC, Van Herle K, Rasgon N, Van Herle A, Martinez D, et al. Verbal memory retrieval deficits associated with untreated hypothyroidism. J Neuropsychiatry Clin Neurosci. 2007;19(2):132-136.

2. Wartofsky L. Myxoedema coma. Endocrinol Metab Clin North Am. 2006;35(4):687-698.

3. Rodriguez I, Fluiters E, Perez-Mendez LF, Luna R, Paramo C, Garcia-Mayor RV. Factors associated with mortality of patients with myxoedema coma: prospective study in 11 cases treated in a single institution. J Endocrinol. 2004;180(2):347-350.

4. Cooper DS, Biondi B. Subclinical thyroid disease. Lancet. 2012;379(9821):1142-1154.

5. Reinhardt W, Mann K. [Incidence, clinical picture and treatment of hypothyroid coma. Results of a survey]. Med Klin (Munich). 1997;92(9):521-524.

6. Wartofsky L, Burman KD. Alterations in thyroid function in patients with systemic illness: the "euthyroid sick syndrome". Endocr Rev. 1982;3(2):164-217.

7. Chaudhari D, Gangadharan V, Forrest T. Heart failure presenting as myxedema coma: case report and review article. Tenn Med. 2013;106(5):39-40.

8. Arvan S. Pericardial tamponade in a patient with treated myxedema. Arch Intern Med. 1983;143(10):1983-1984.

9. Dattilo G, Crosca S, Tavella S, Marte F, Patane S. Pericardial effusion associated with subclinical hypothyroidism. Int J Cardiol. 2011;153(3):e47-50. 